Case report

\title{
A Case in which Laparoscopic Bilateral Salpingo- Oohorectomy was Indispensable due to Bilateral Tubo-Ovairan Abscesses in a Young Woman
}

\author{
Satoru Takeuchi \\ Kochi Health Sciences Center, Gynecology and Obstetrics, Kochi, Japan
}

\begin{abstract}
Tubo-ovarian abscess (TOA) requires admission to hospital, i.v. antibiotics and, possibly, aspiration or surgery. A 35-year-old woman who was G2P1 was brought into the emergency department because of acute abdomen. A transvaginal ultrasonography demonstrated bilateral cystic adnexal tumors and a moderate amount of ascites suspected to be puss. Laparoscopic examination revealed that the left and right adnexa each formed an inflammatory mass. The left and right tubo-ovarian abscesses both formed masses, and the ovaries were indistinguishable from the tubes and other inflammatory tissues. Laparoscopic bilateral salpingo-oophorectomy was indispensable. Bacterial culture showed no bacterial growth. Performance of laparoscopic bilateral salpingo-oophorectomy was indispensable due to bilateral tubo-ovarian abscesses in this patient.
\end{abstract}

Key words: tubo-ovarian abscess, bilateral salpingo-oophorectomy (J Rural Med 2009; 4(2): 72-74)

\section{Introduction}

Pelvic inflammatory disease (PID) is a common infection among reproductive-aged women ${ }^{1)}$. Tubo-ovarian abscess (TOA) represents a severe form of PID ${ }^{1)}$. TOA requires admission to hospital, i.v. antibiotics and, possibly, aspiration or surgery ${ }^{2}$. If antibiotic therapy is insufficient, operative intervention is performed in patients with TOA. Performance of laparoscopic bilateral salpino-oophorectomy was indispensable due to bilateral tubo-ovarian abscesses in this patient.

Correspondence to: Satoru Takeuchi

Kochi Health Sciences Center, Gynecology and Obstetrics, Ike 2125-1, Kochi City, Kochi 781-8555, Japan

E-mail: satoru_takeuchi@khsc.or.jp

\section{Case Presentation}

A 35-year-old woman who was G2P1 was brought into the emergency department because of acute abdomen lasting for several hours. Her past medical history was unremarkable except for PID fourteen years previously. On physical examination, the patient had severe lower abdominal pain and was febrile, with a regular pulse of 90 beats/minute and blood pressure of $116 / 70 \mathrm{mmHg}$. Lower abdominal tenderness was noted. Her body temperature was $38.8^{\circ} \mathrm{C}$. Laboratory examination revealed a CRP of $7.80 \mathrm{mg} / \mathrm{dl}$, WBC of $16,500 / \mu \mathrm{l}$, neutrophil differential of $89.3 \%$ and $\mathrm{Hb}$ of $8.4 \mathrm{~g} / \mathrm{dl}$. CT examination revealed pelvic cystic masses (Figure 1). The patient was referred to the gynecology department. A transvaginal ultrasonography demonstrated bilateral cystic adnexal tumors and a moderate amount of ascites suspected to be puss (Figures 2, 3 and 4). The patient was diagnosed as having bilateral tubo-ovarian abscesses and an emergency operation was performed.

A laparoscopic camera was introduced via a $12-\mathrm{mm}$ umbilical port. Laparoscopic examination revealed that the left and right adnexa each formed an inflammatory mass that was adhered to the broad ligaments. In the process of separating the inflammatory masses, whitish green pus was observed flowing from the masses. Culture of the puss was performed. The ovaries were indistinguishable from tubes and other inflammatory tissues. Performance of laparoscopic bilateral salpingo-oophorectomy was indispensable. There were any other inflammatory lesions in the abdominal cavity. The abdomen was sufficiently washed with saline solution. A Penrose drainage tube was inserted into the culde-sac. The postoperative course was uneventful. Bacterial culture showed no bacterial growth. The patient was discharged on the sixth day after the operation. Two weeks after the operation, Chlamydia PCR was performed on a sample collected at the cervical canal, and the result was pos- 


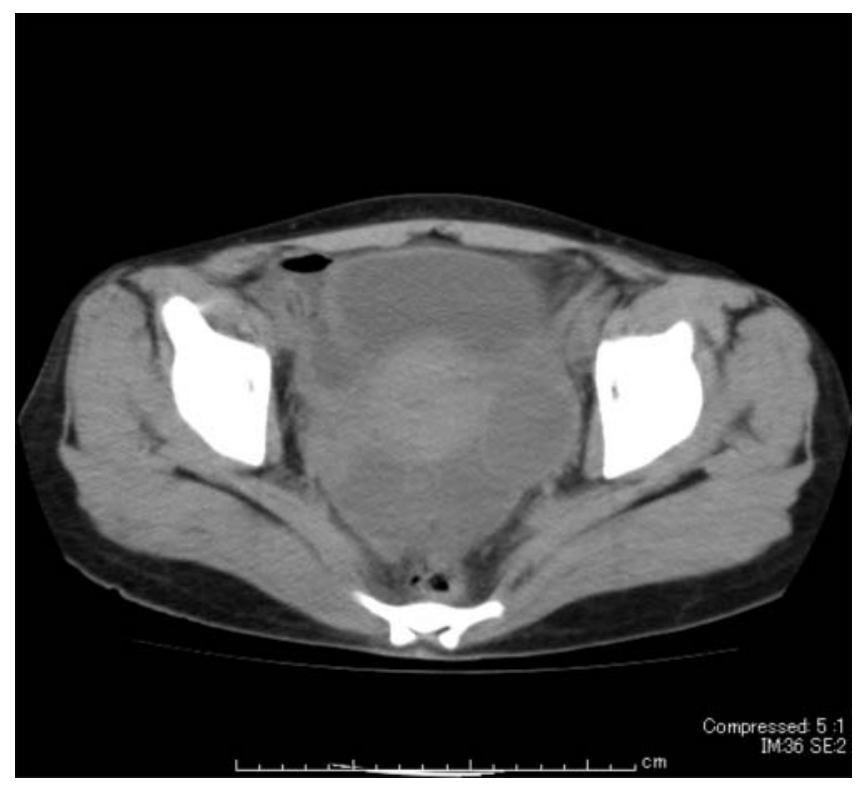

Figure 1 CT image of tubo-ovarian abscesses.

itive. The patient was prescribed macrolide antibiotics.

\section{Discussion}

Gynecologic emergencies are relatively common and include ectopic pregnancies, adnexal torsion, tubo-ovarian abscess, hemorrhagic ovarian cysts, gynecologic hemorrhage and vulvovaginal trauma ${ }^{3}$. PID and upper genital tract infection describe inflammatory changes in the upper female genital tract of any of the following combinations: endometritis, salpingitis, tubo-ovarian abscess and peritonitis in the small pelvis. In cases of pelvic infections that the bacteria passes through a uterus and tubes, Chlamydia trachomatis and Neisseria gonorrhea are common, with increasing incidences ${ }^{4}$. Adhikari et al. reported the age at occurrence of tubo-ovarian abscess ranges from 14 to 45 years (mean 27 years) $)^{2}$. Seven (35\%) patients in their report had a prior history of PID or sexually transmitted disease, and $1(5 \%)$ was febrile ${ }^{2}$. All had lower abdominal tenderness, and $9(45 \%)$ had cervical motion or adnexal tenderness. Sonographic abnormalities included 14 patients $(70 \%)$ with a complex adnexal mass, $5(25 \%)$ with echogenic fluid in the cul-de-sac and $3(15 \%)$ with pyosalpinx ${ }^{2}$. The present case had tubo-ovarian abscesses with a prior history of PID, lower abdominal tenderness and a sonographic abnormality in the form of complex adnexal masses with echogenic fluid in the cul-de-sac; she was also febrile. The patient had almost all of the symptoms reported by Adhikari et al. Bacterial culture showed no bacterial growth however, histopathologic

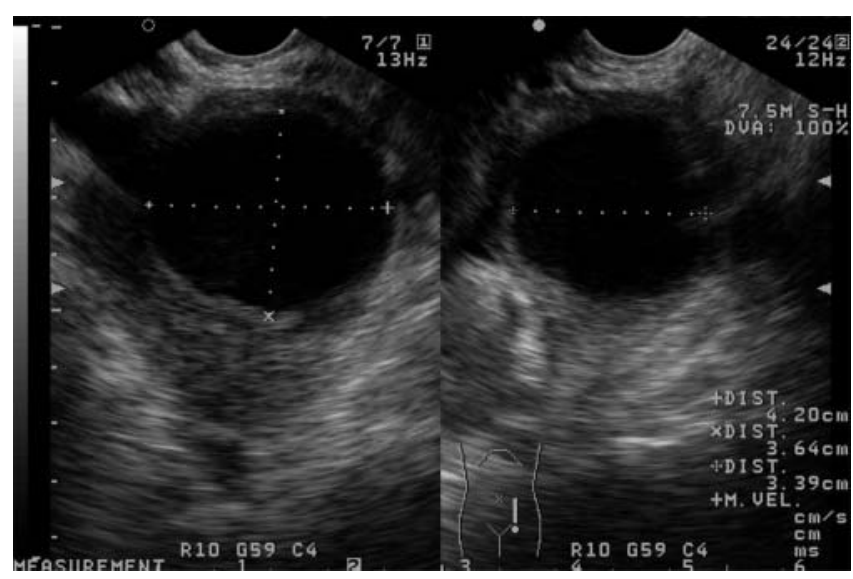

Figure 2 Transvaginal ultrasonographic image of the left tuboovarian abscess.

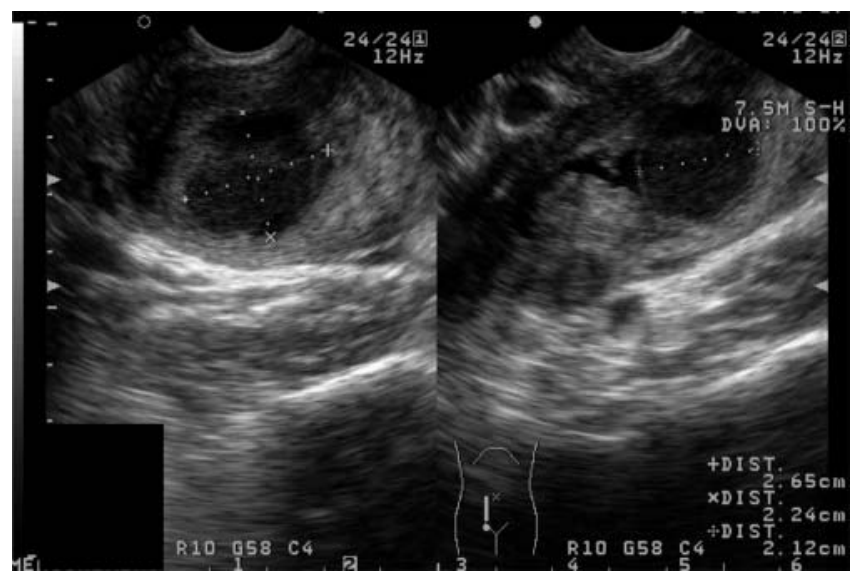

Figure 3 Transvaginal ultrasonographic image of the right tuboovarian abscess.

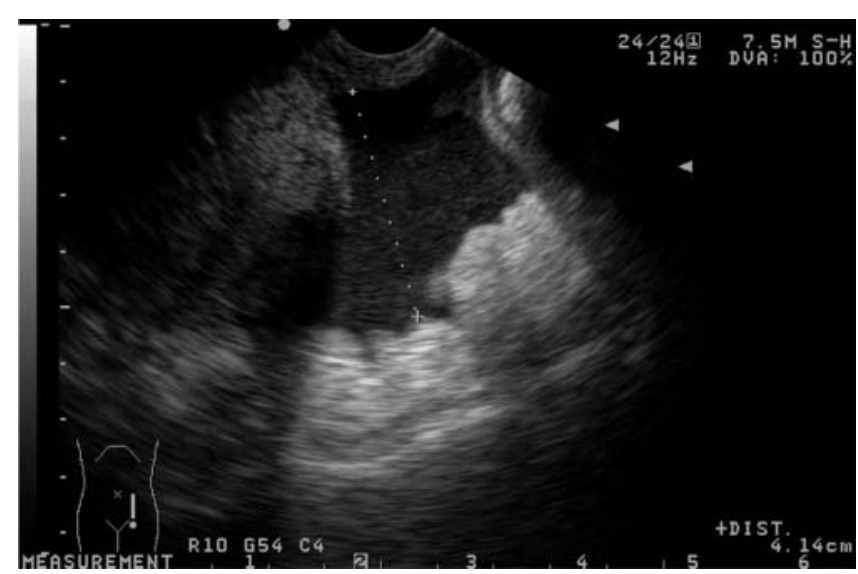

Figure 4 Transvaginal ultrasonographic image of puss at the Douglas pouch 
examination of the resected specimen revealed chronic salpingitis and oophoritis in both adenexae. It showed that the fluid in the pelvic mass was puss as presumed in the macroscopic findings.

Laparoscopic surgery is considered to be a less invasive surgery than laparotomy. Advanced laparoscopic procedures are increasingly being used as alternatives to laparotomy in gynecologic surgery. Intrinsically, laparoscopic surgery has the characteristics of microscopic surgery and has the benefit of making it easy to perform meticulous procedures. An operator looks at the operating site from a distance of about $30-50 \mathrm{~cm}$ during a laparotomy procedure. On the other hand, a laparoscope can reach any distance from the operation site and magnify the target tissue, and the operator can see behind the targeted organs. In the present case, it was noted that each tubo-ovarian abscess was in a mass and that the ovaries were indistinguishable from tubes and other inflammatory tissues during separation of the ovaries from other. However, laparoscopic surgery was associated with improved cosmesis and faster return to normal daily activities because it causes fewer pains than laparotomy.

Performance of laparoscopic bilateral salpino-oophorectomy was indispensable due to bilateral tubo-ovarian abscesses in this patient.

\section{References}

1. Lareau SM, Beigi RH. Pelvic inflammatory disease and tuboovarian abscess. Infect Dis Clin North Am 2008; 22: 693708.

2. Adhikari S, Blaivas M, Lyon M. Role of bedside transvaginal ultrasonography in the diagnosis of tubo-ovarian abscess in the emergency department. Emerg Med 2008; 34: 429-433.

3. McWilliams GD, Hill MJ, Dietrich CS 3rd. Gynecologic emergencies. Surg Clin North Am 2008; 88: 265-283.

4. Hoof K. Pelvic inflammatory disease. Ther Umsch 2007; 64: 365-368. 